\title{
Prevalence and Significance of Exit Block During Arrhythmias Arising in Pulmonary Veins
}

\author{
HUNG-FAT TSE, M.D., CHU-PAK LAU, M.D., WILLIAM KOU, M.D.,* \\ FRANK PELOSI, M.D.,* HAKAN ORAL, M.D.,* MICHAEL KIM, M.D.,* \\ GREGORY F. MICHAUD, M.D.,* BRADLEY P. KNIGHT, M.D.,** \\ MAURO MOSCUCCI, M.D.,* S. ADAM STRICKBERGER, M.D.,* \\ and FRED MORADY, M.D.*
}

From the Division of Cardiology, Department of Medicine, University of Hong Kong, Queen Mary Hospital, Hong Kong; and the *Division of Cardiology, Department of Internal Medicine, University of Michigan Medical Center, Ann Arbor, Michigan

Exit Block. Introduction: Recent studies described the occurrence of conduction block within pulmonary veins. The purpose of this study was to evaluate the prevalence of exit block during arrhythmias that arise in pulmonary veins.

Methods and Results: Twenty-five patients with atrial tachycardia/fibrillation underwent successful ablation of 28 arrhythmogenic foci within a pulmonary vein. The prevalence of exit block in the pulmonary veins was determined in 28 arrhythmogenic pulmonary veins and 40 nonarrhythmogenic pulmonary veins. During isolated premature depolarizations, exit block in a pulmonary vein was observed at $50 \%$ of arrhythmogenic pulmonary vein sites and was never observed within pulmonary veins that did not generate a tachycardia $(P<0.01)$. During tachycardia, exit block from a pulmonary vein was observed in $61 \%$ of the arrhythmogenic pulmonary veins. The mean cycle length of the pulmonary vein tachycardias associated with exit block was significantly shorter than the cycle length of tachycardias that were not associated with exit block $(163 \pm 32$ vs $251 \pm 45 \mathrm{msec}$, $\mathrm{P}<0.001$ ). Exit block in two pulmonary veins during the same episode of tachycardia was observed in 3 of the 28 arrhythmogenic pulmonary veins $(11 \%)$ in three different patients. Simultaneous recordings in the two pulmonary veins demonstrated bursts of tachycardia in both veins that were not synchronized. Radiofrequency catheter ablation of the arrhythmogenic site in one of the pulmonary veins eliminated spontaneous recurrences of tachycardia from the other pulmonary vein.

Conclusion: Exit block from pulmonary veins is a common observation during tachycardias generated within pulmonary veins and indicates that an arrhythmogenic pulmonary vein has been identified. The occurrence of exit block in more than one pulmonary vein most likely is attributable to simultaneous tachycardias, one or both of which may be tachycardia induced and perpetuated by the other. (J Cardiovasc Electrophysiol, Vol. 11, pp. 379-386, April 2000)

pulmonary vein, exit block, radiofrequency ablation

\section{Introduction}

Recent studies demonstrated that pulmonary veins have complex electrophysiologic properties and that there may be multiple mechanisms by which pulmonary vein foci either trigger or generate atrial arrhythmias. ${ }^{1-5}$ Conduction block of depolarizations arising in the pulmonary veins has been described. ${ }^{2-4}$ However, the prev-

Address for correspondence: Fred Morady, M.D., Division of Cardiology, B1F245, University of Michigan Medical Center, 1500 East Medical Center Drive, Ann Arbor, MI 48109-0022. Fax: 734-9367026.

Manuscript received 10 September 1999; Accepted for publication 30 December 1999. alence and clinical significance of this finding are unclear. Therefore, the purpose of this study was to evaluate the prevalence of exit block during arrhythmias that arise in pulmonary veins. We also sought to clarify the prevalence and mechanism of exit block in two pulmonary veins during the same episode of tachycardia.

Methods

\section{Patient Population}

The study population consisted of 25 consecutive patients with symptomatic atrial tachycardia/fibrillation who underwent successful radiofrequency catheter ablation of an arrhythmogenic site within a pulmonary vein. There were 19 men and 6 women (mean age \pm SD: $44 \pm$ 
13 years). None of the patients had structural heart disease. Mean left ventricular ejection fraction was $0.63 \pm$ 0.06 , and mean left atrial diameter determined by echocardiography was $3.7 \pm 0.4 \mathrm{~cm}$. Clinically documented arrhythmia was atrial tachycardia in 3 patients, paroxysmal atrial fibrillation in 19 , and persistent atrial fibrillation in 3. Except in the patients who had persistent atrial fibrillation, 24-hour ambulatory ECG recordings demonstrated frequent atrial premature depolarizations and runs of nonsustained atrial tachycardia and/or atrial fibrillation. The patients had been symptomatic for a mean of $42 \pm 36$ months. Treatment with a mean of $4 \pm 2$ antiarrhythmic drugs had been ineffective. Three of the patients in this study also were included in a report describing the results of focal ablation of atrial fibrillation. ${ }^{3}$

In another eight patients, localization and ablation of an arrhythmia thought to be arising in a pulmonary vein was unsuccessful because of an inability to localize the site of origin of the arrhythmia, because of the presence of multiple foci, or because of the absence of spontaneous arrhythmias during the procedure. Data from these patients were not included in this study.

For the purposes of this study, a successful outcome was defined as elimination of arrhythmias that had been arising in a pulmonary vein during at least 60 minutes of observation in the electrophysiology laboratory and at least 3 months of follow-up.

\section{Electrophysiologic Procedures}

Electrophysiologic procedures were performed with patients in the fasting state after informed consent was obtained. All antiarrhythmic drug therapy was discontinued at least five half-lives before the procedure. Three multipolar electrode catheters were introduced through a femoral or subclavian vein. One catheter was positioned in the high lateral right atrium, one in the coronary sinus, and a 6- or 7-French, quadripolar, ablation catheter with a thermistor embedded in a 4-mm distal electrode (EP Technologies, Sunnyvale, CA, USA) was positioned near the His bundle and later used for mapping and ablation. Surface ECG leads and bipolar intracardiac electrograms were filtered at 30 to $500 \mathrm{~Hz}$ and recorded on paper or optical disk. Pacing was performed at twice diastolic threshold using stimuli of 2-msec duration.

\section{Mapping and Ablation}

During the procedure, the patients in this study either had (1) frequent atrial premature depolarizations and/or runs of nonsustained atrial fibrillation/tachycardia that were spontaneous or induced by pacing or isoproterenol infusion, or (2) early reinitiation of spontaneous atrial fibrillation after internal or external cardioversion. Internal or external cardioversion was performed during the procedure if an episode of spontaneous or induced atrial fibrillation persisted for more than 10 minutes. The technique used for internal cardioversion has been described previously. ${ }^{6}$
Mapping of the left atrium and pulmonary veins was performed after initial mapping in all patients indicated that the site of origin of the atrial arrhythmias was not in the right atrium, superior vena cava, or coronary sinus. After transseptal catheterization, a 3,000- to $5,000-\mathrm{U}$ bolus of heparin was administrated, followed by an additional 1,000-U bolus every hour. Positioning of the ablation catheter within a pulmonary vein was verified in all patients by the fluoroscopic appearance of the ablation catheter outside of the cardiac silhouette on multiple views. In each patient, both the left and right superior pulmonary veins were mapped. The left and right inferior pulmonary veins were mapped in 10 and 8 patients, respectively. In 7 patients, two electrode catheters were inserted into the left atrium and positioned within the left and right superior pulmonary veins to allow simultaneous mapping in the two veins.

Ablation was performed at sites of earliest activation during premature depolarizations that initiated atrial tachycardia or fibrillation. Radiofrequency energy was delivered at a cycle length of 500 to $550 \mathrm{kHz}$ for 30 to 120 seconds by a generator that automatically adjusted the output to maintain a preset temperature of $52^{\circ}$ to $60^{\circ} \mathrm{C}$ at the electrode-tissue interface (EP Technologies; or Medtronic Inc., Minneapolis, MN, USA). A mean of $1.8 \pm 2.0$ applications (range 1 to 5 ) of radiofrequency energy was delivered in arrhythmogenic pulmonary veins.

A total of 28 pulmonary vein foci were successfully ablated in 25 patients. Fourteen were located in the left superior pulmonary vein, 12 in the right superior pulmonary vein, and 2 in the left inferior pulmonary vein. Mean duration of fluoroscopy was $60 \pm 41$ minutes (range 22 to 109). The only complication was a small pericardial effusion that subsided with conservative treatment in two patients.

\section{Electrogram Analysis}

Exit block was defined as the occurrence of spontaneous depolarizations within a pulmonary vein that failed to propagate on a 1:1 basis to the right atrium and coronary sinus. With the proximal pair of electrodes of the mapping catheter positioned at the ostium of the pulmonary vein, the site of exit block was classified as either (1) within the pulmonary vein, between the distal and proximal electrode pairs (Fig. 1A), or (2) at the ostium, between the proximal pair of electrodes and the rest of the atria (Fig. 1B). Endocardial activation times were measured relative to the high right atrial electrogram.

\section{Statistical Analysis}

Continuous variables are expressed as mean $\pm 1 \mathrm{SD}$ and compared using Student's $t$-test or by analysis of variance, as appropriate. Discrete variables were compared with the Fisher exact test. $\mathrm{P}<0.05$ was considered statistically significant. 
A

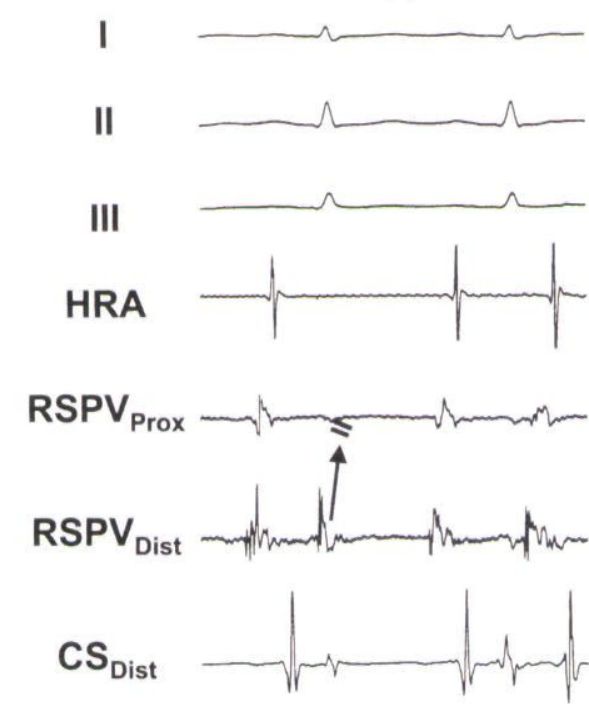

B

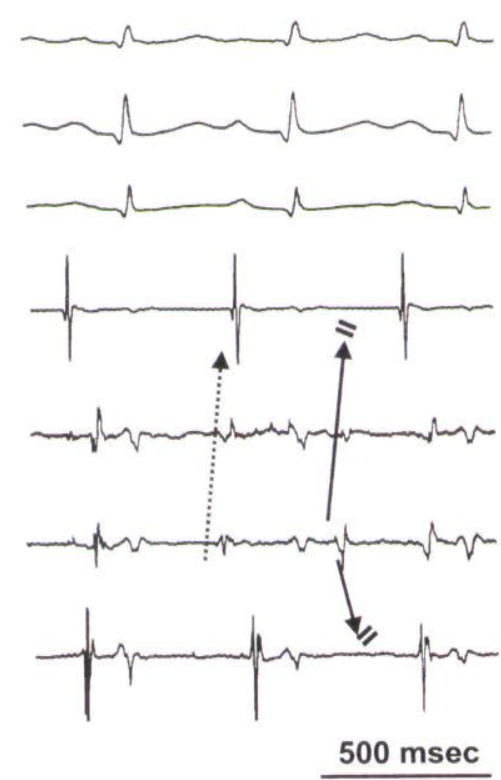

Figure 1. Examples of different sites of exit block during premature depolarizations in arrhythmogenic pulmonary veins. In these examples, the proximal pair of electrodes of the ablation catheter was positioned at the ostium of the right superior pulmonary vein (RSPV $\left.V_{P r o x}\right)$ and the distal pair $\left(R S P V_{\text {Dist }}\right)$ was positioned approximately $1 \mathrm{~cm}$ into the vein. (A) Burst of premature depolarizations recorded in the right superior pulmonary vein, with an episode of block between the distal and proximal electrode pairs. (B) Two consecutive premature depolarizations with coupling intervals of 360 and $340 \mathrm{msec}$, recorded in the right superior pulmonary vein. The first one propagates to the atrium and fuses with the sinus beat, causing a change in $P$ wave morphology. The second one propagates to the proximal portion of the pulmonary vein, but not to the rest of the atria. CS ${ }_{D i s t}=$ recordings by the distal pair of electrodes of the coronary sinus catheter; HRA = high right atrial electrogram.

\section{Results}

\section{Follow-Up}

After a mean follow-up period of $9 \pm 5$ months (range 3 to 19), 19 patients had no symptomatic arrhythmias in the absence of antiarrhythmic drug therapy and 6 patients had a recurrence of atrial tachycardia/fibrillation that was found during repeat electrophysiologic testing to be arising from a pulmonary vein other than the one that contained the original target site.

\section{Exit Block During Premature Depolarizations}

Depending on the coupling interval of the premature depolarization recorded in the pulmonary vein, there was either exit block (Fig. 1A), a global atrial depolarization, or a fusion complex with a sinus beat (Fig. 1B). Exit block during premature depolarizations was observed in $14(50 \%)$ of the 28 arrhythmogenic pulmonary veins. In the pulmonary veins that did not generate a tachycardia, spontaneous premature depolarizations were not observed, and there were no instances of exit block $(0 / 40$, $\mathrm{P}<0.01$ compared to $50 \%$ prevalence of exit block in arrhythmogenic pulmonary veins). The coupling interval of premature depolarizations associated with exit block was significantly shorter than that of the premature depolarizations associated with global atrial activation (185 \pm 42 vs $240 \pm 43 \mathrm{msec}, \mathrm{P}<0.05)$.

\section{Exit Block During Tachycardias}

Exit block was observed during 17 (61\%) of 28 pulmonary vein tachycardias. In $13(76 \%)$ of these 17 tachycardias, the exit block occurred at the onset of tachycardia (Fig. 2). In the remaining 4 (24\%) of 17 tachycardias that displayed exit block, the exit block occurred several seconds or minutes after the onset of tachycardia (Fig. 3). The mean cycle length of the tachycardias associated with exit block was significantly shorter than that of the tachycardias not associated with exit block ( $163 \pm 32$ vs $251 \pm 45 \mathrm{msec}, \mathrm{P}<0.001$ ) (Fig. 4).

Exit block was noted within the pulmonary vein, between the distal and proximal pairs of electrodes, in 9 $(53 \%)$ of the 17 tachycardias that displayed exit block, and at the pulmonary vein ostium in $8(47 \%)$ of the 17 tachycardias. Among the 17 pulmonary vein tachycardias that exhibited exit block, the site of origin was in the right superior pulmonary vein in $10(59 \%)$, the left superior pulmonary vein in $6(35 \%)$, and the left inferior pulmonary vein in $1(6 \%)$.

\section{Exit Block in Two Pulmonary Veins}

In $3(11 \%)$ of the 28 pulmonary vein tachycardias in three different patients, exit block was observed in two pulmonary veins during the same episode of tachycardia. In two of these patients, recordings were obtained se- 


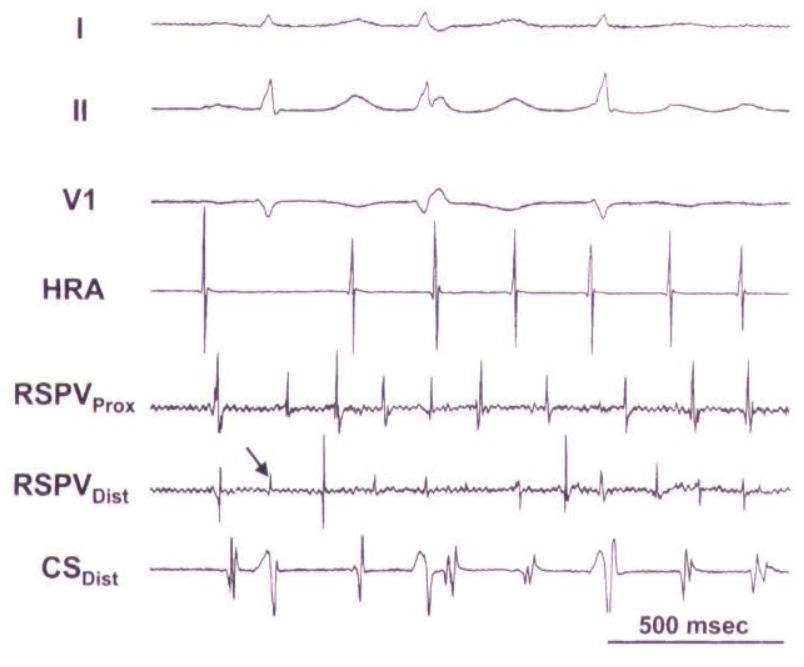

Figure 2. Example of rate-related exit block to the atrium at the onset (arrow) of a tachycardia that originated in the right superior pulmonary vein $(R S P V)$. The average cycle length in the high right atrium (HRA) and distal coronary sinus $\left(C S_{\text {Dist }}\right)$ is $190 \mathrm{msec}$, compared with an average cycle length of $120 \mathrm{msec}$ in the pulmonary vein.

quentially from the two pulmonary veins with a single mapping catheter. These two patients had frequent spontaneous episodes of nonsustained atrial fibrillation sepa- rated by only one to two sinus beats. Exit block was observed in the left and right superior pulmonary veins in one of the patients, and in the left superior and inferior pulmonary veins in the other patient. In both patients, activation within both of the pulmonary veins that displayed exit block progressed from the distal to proximal pair of electrodes, with exit block to the rest of the atria. One or two applications of radiofrequency energy in the left superior pulmonary vein restored sinus rhythm and eliminated recurrences of atrial fibrillation in both patients. Although no applications of radiofrequency energy were delivered in the other pulmonary vein that had displayed exit block, there were no further spontaneous episodes of atrial fibrillation in either patient after delivery of radiofrequency in the left superior pulmonary vein.

In a third patient in whom exit block was observed in two pulmonary veins, two mapping catheters were placed in the left atrium, allowing simultaneous recordings in the two pulmonary veins. Exit block, with activation progressing from the distal to proximal pair of electrodes, was observed in both the left and right superior pulmonary veins during a sustained episode of atrial fibrillation that had been present for several weeks. Simultaneous recordings in the left and right superior pulmonary veins demonstrated that the electrograms arising

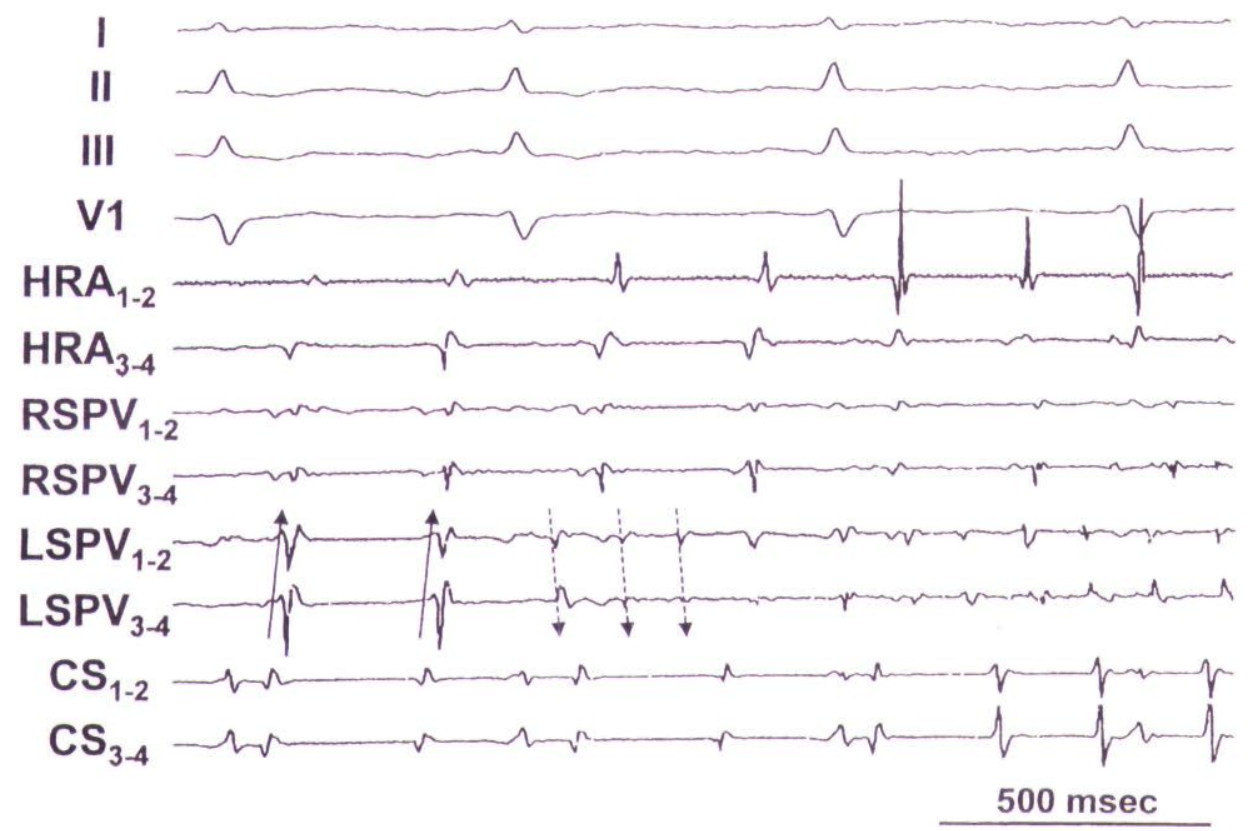

Figure 3. Example of exit block to the atrium of a tachycardia that originated in the left superior pulmonary vein (LSPV), in the presence of simultaneous atrial fibrillation. In this case, a single depolarization (not shown) originating in the LSPV initiated an episode of atrial fibrillation that has a mean cycle length of $280 \mathrm{msec}$ in the atrium. During atrial fibrillation, passive activation in the LSPV and right superior pulmonary vein $(R S P V)$ is observed, with activation progressing from the proximal ( $L S P V_{3-4}$ and $R S P V_{3-4}$ ) to the distal pair (LSPV 1.2 and RSPV 1.2 ) of electrodes (arrow). Five seconds later, a tachycardia originating in the LSPV that has a mean cycle length of 120 msec is observed. During this tachycardia, activation within the pulmonary vein progresses from the distal ( LSPV 1-2 $_{2}$ to the proximal pair $\left(L S P V_{3-4}\right)$ of electrodes (dotted arrow). Exit block occurs as a result of rate-related conduction block, with failure of 1:1 activation of the atrium. Note that the mean cycle length of atrial fibrillation

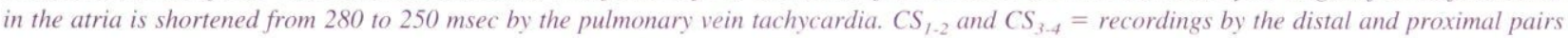
of electrodes of the coronary sinus catheter, respectively; $H R A_{1-2}$ and $H R A_{3-4}=$ recordings by the distal and proximal pairs of electrodes of the high right atrial catheter, respectively. 


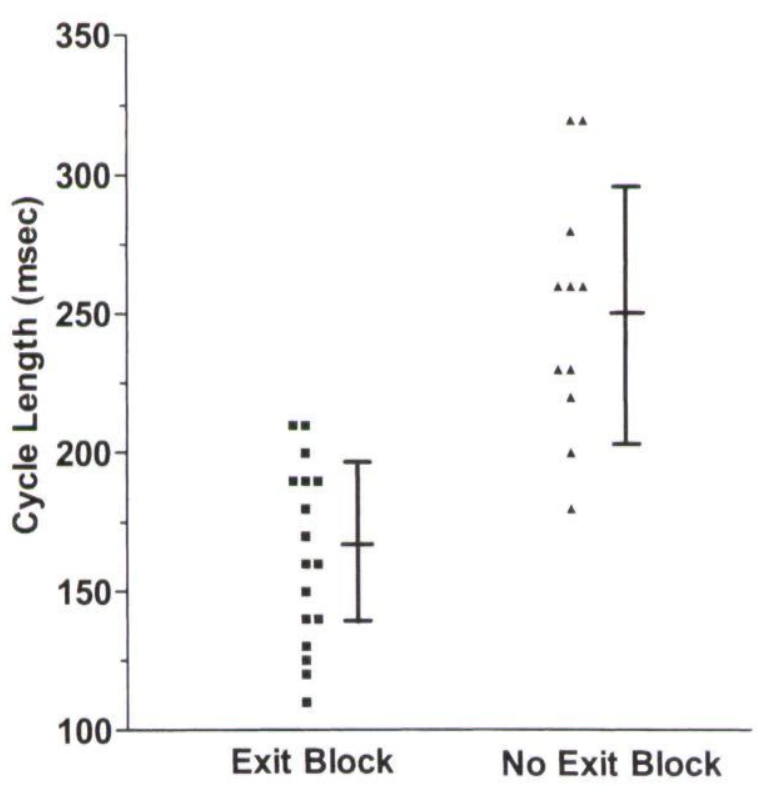

Figure 4. Comparison of the mean cycle length of tachycardias recorded in 17 arrhythmogenic pulmonary veins that displayed exit block and 11 arrhythmogenic pulmonary veins that did not display exit block. Mean $\pm 1 S D$ also is shown for each group. Mean tachycardia cycle length was significantly shorter when there was exit block than when there was not $(P<0.001)$.

in the two veins were not synchronized. There were intermittent bursts of rapid activity, separated by interspersed periods of passive activation from the atria (Fig. 5A). An application of radiofrequency energy in the left superior pulmonary vein during atrial fibrillation restored sinus rhythm (Fig. 5B). Although there were no further spontaneous arrhythmias, a nonsustained tachycardia arising in the right superior pulmonary vein was inducible by rapid atrial pacing (Fig. 5C). After one more application of radiofrequency energy in the right superior pulmonary vein, there were no further spontaneous or inducible arrhythmias (Fig. 5D).

\section{Discussion}

\section{Main Findings}

The results of this study demonstrate that exit block from pulmonary veins is a common finding, occurring during $>50 \%$ of tachycardias arising in pulmonary veins. The exit block is attributable to rate-related conduction block out of a pulmonary vein. The simultaneous occurrence of exit block in more than one pulmonary vein during atrial fibrillation appears to be attributable to simultaneous pulmonary vein tachycardias, one of which may be triggered by the other.

The frequent occurrence of exit block from pulmonary veins during atrial fibrillation suggests that the pulmonary veins often may be more than simply the source of a premature depolarization that triggers atrial fibrillation. Based on the observation that atrial fibrillation may terminate shortly after the delivery of radiofre- quency energy to an arrhythmogenic site in a pulmonary vein, it appears that the bursts of tachycardia associated with exit block from pulmonary veins may be important in sustaining the atrial fibrillation.

\section{Conduction Properties of Pulmonary Veins}

The frequent occurrence of exit block from pulmonary veins during premature depolarizations and during tachycardia may be attributable to the complex arrangement of myocardial fibers at the junction between the pulmonary veins and the left atrium. Previous pathologic studies showed that these junctions are formed by extensions of myocardial fibers from the atrial wall running in an oblique or spiral direction into the pulmonary veins. ${ }^{7}$ It is possible that the complex anatomic arrangement of these fibers within the pulmonary veins results in discontinuous propagation of wavefronts, predisposing to exit block. $^{8-11}$

\section{Mechanism of Exit Block}

In this study, exit block in arrhythmogenic pulmonary veins was found to be a rate-related phenomenon. In the majority of cases, exit block was observed at the onset of tachycardia that had a short cycle length, resulting in conduction block to the rest of the atria. Less commonly, a tachycardia arising in a pulmonary vein resulted in induction of simultaneous atrial fibrillation, and exit block occurred several seconds to minutes after the onset of tachycardia. In these cases, the cycle length of the pulmonary vein tachycardia accelerated and was shorter than that of the atrial fibrillation, resulting in failure of $1: 1$ conduction out of the pulmonary vein. Regardless of whether the exit block is observed at the onset of a pulmonary vein tachycardia or after the tachycardia has already been present for several seconds or more, the occurrence of exit block suggests the presence of an arrhythmogenic focus in that particular vein.

\section{Exit Block in Two Pulmonary Veins}

The occurrence of exit block in two pulmonary veins during a given episode of atrial fibrillation can be explained by a single site of origin of a tachycardia and an insulated connection between the two pulmonary veins. This mechanism would explain why an application of radiofrequency energy in only one of the two veins was sufficient to restore sinus rhythm and abolish spontaneous recurrences of atrial fibrillation in the three patients in this study in whom exit block was observed in more than one pulmonary vein. If there were an insulated connection between two pulmonary veins, simultaneous recordings from the two pulmonary veins might be expected to demonstrate a synchronized pattern of electrograms. However, among the three patients in this study in whom exit block was observed in two pulmonary veins, simultaneous recordings from both of the pulmonary veins were available in one patient. In this patient, the tachycardias recorded at the same time in the two 


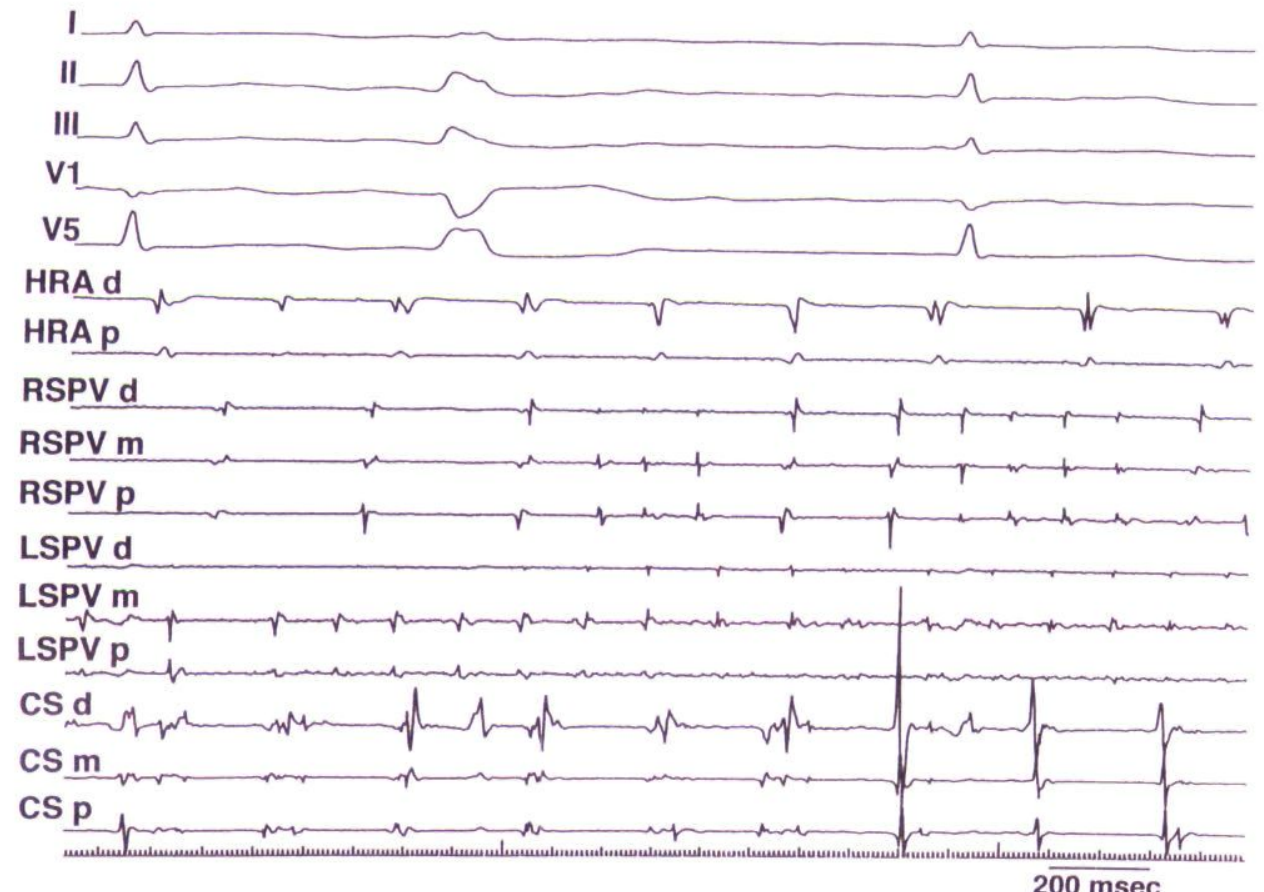

A

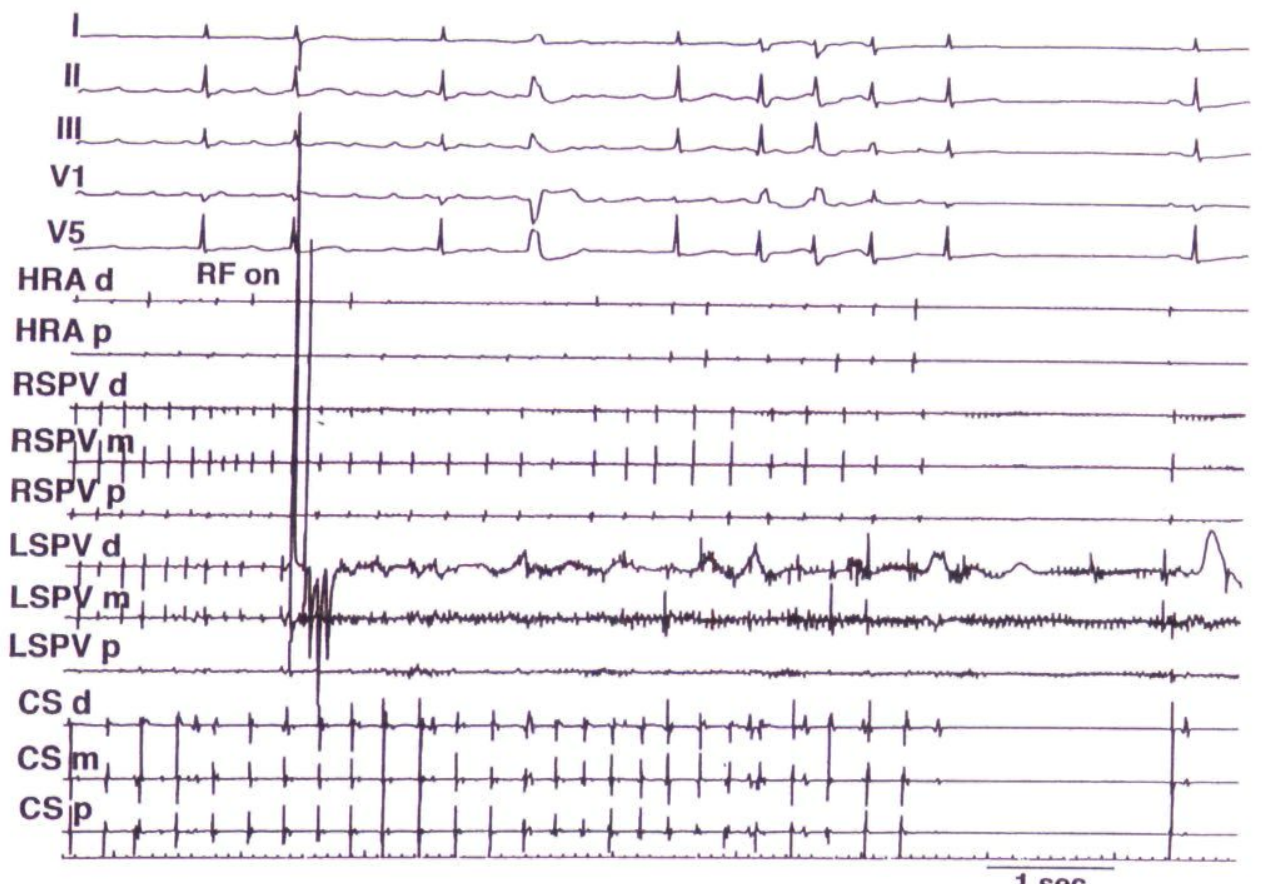

Figure 5. Example of simultaneous exit block in the left and right superior pulmonary veins. (A) During atrial fibrillation, there are frequent short bursts of tachycardia that result in exit block in both the left and right superior pulmonary veins. Note that the bursts of tachycardia are not synchronized to each other. (B) Termination of atrial fibrillation by an application of radiofrequency $(R F)$ in the left superior pulmonary vein. Although no applications of $R F$ energy were delivered in the right superior pulmonary vein, there were no more spontaneous arrhythmias. (C) Although there were no more spontaneous arrhythmias arising from the right superior pulmonary vein, a nonsustained tachycardia associated with exit block in the right superior pulmonary vein was inducible by rapid atrial pacing. Activation within the left superior pulmonary vein occurred passively, from the atrium. (D) After mapping and ablation within the right superior pulmonary vein, tachycardia arising in a pulmonary vein was no longer inducible. Only short episodes of nonsustained atrial fibrillation with passive activation of the left and right pulmonary veins could be induced. Abbreviations as in previous figures. 

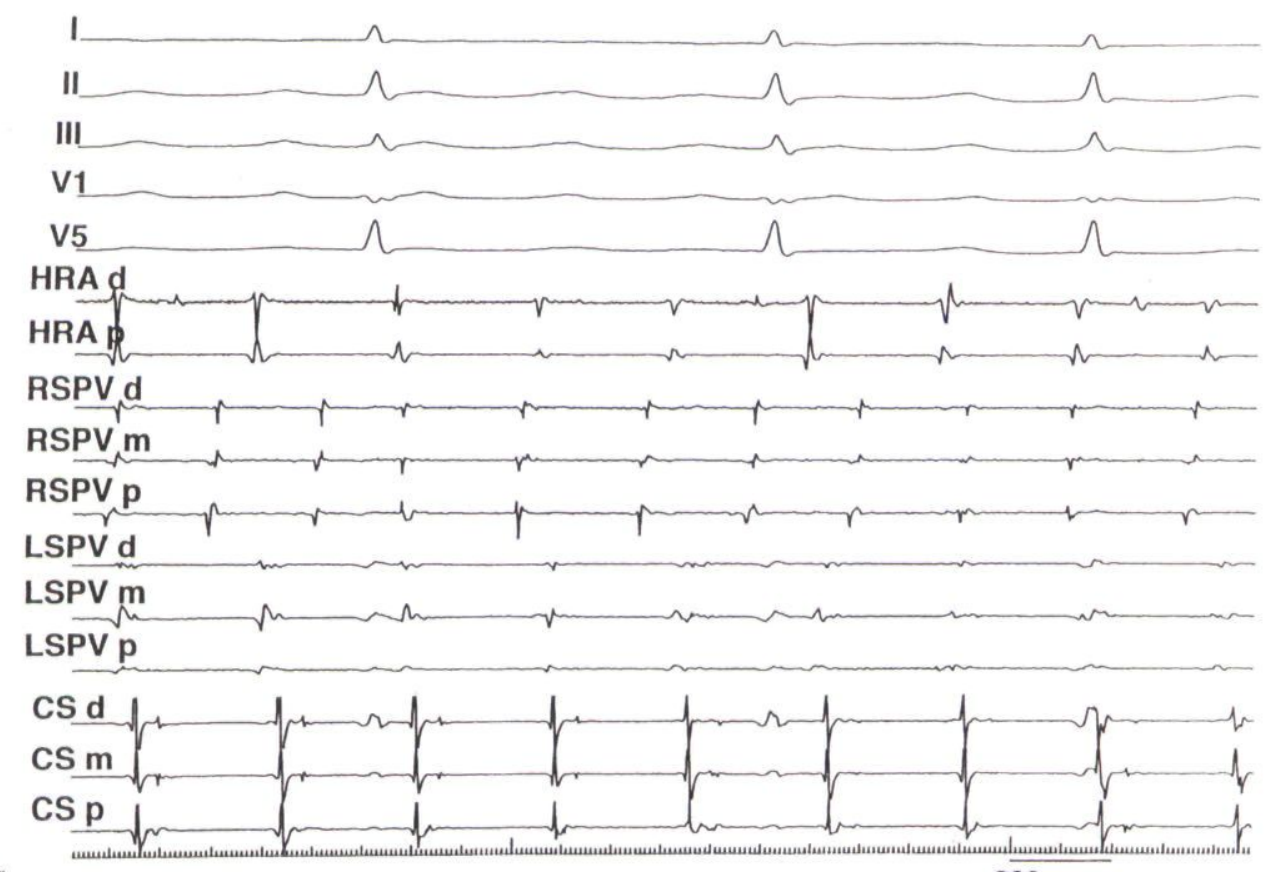

C

$200 \mathrm{msec}$

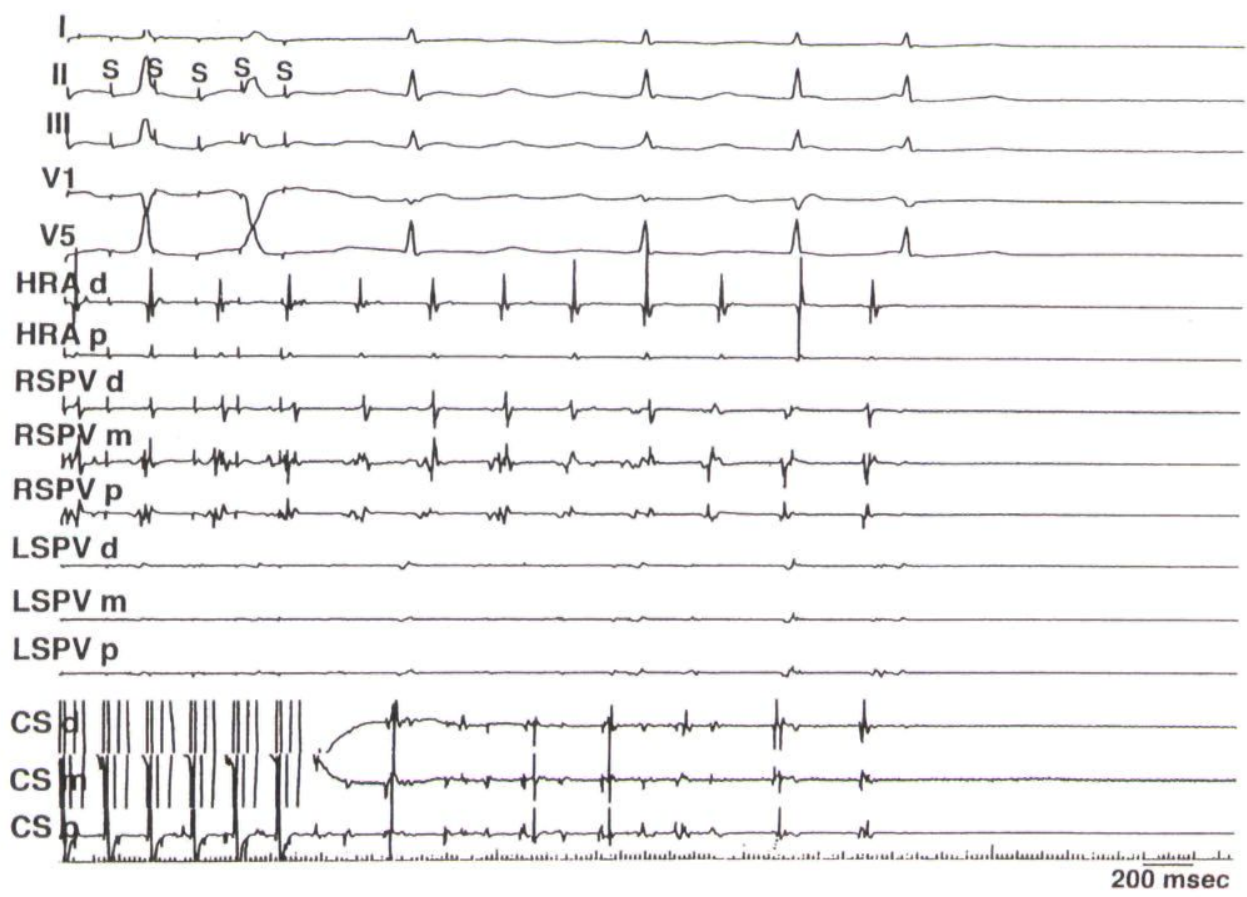

Figure 5. (continued)

pulmonary veins were not synchronized to each other. This finding does not support the presence of an insulted connection between pulmonary veins.

The observation of nonsynchronized bursts of tachycardia with exit block in two pulmonary veins suggests that tachycardias may be generated at the same time in more than one pulmonary vein. Based on the observations that spontaneous arrhythmias resolved after ablation in only one of the two pulmonary veins and that tachycardia in the pulmonary vein that was not targeted for ablation still was inducible by rapid pacing, it seems likely that tachycardia arising in one of the pulmonary veins was repeatedly triggering bursts of tachycardia in another pulmonary vein. Therefore, once the tachycardia generated in one of the arrhythmogenic pulmonary veins has been eliminated by ablation, the trigger for the tachycardia arising in the other pulmonary vein also may be eliminated. 


\section{Prior Studies}

A prevalence of exit block of approximately $50 \%$ in arrhythmogenic pulmonary veins is consistent with the $34 \%$ to $47 \%$ prevalence reported in previous studies. ${ }^{2,4}$ In accordance with the results of the present study, Chen et al. ${ }^{4}$ demonstrated that exit block is most likely to occur in the right superior pulmonary. They postulated that this may be attributable to a more complex arrangement of myocardial fibers with a greater anisotropic conduction properties in the right superior pulmonary vein than in the left superior pulmonary vein, and a significant difference in atrial effective refractory period between the proximal and distal right superior pulmonary vein. Furthermore, as shown by Haïssaguerre et $\mathrm{al}^{2}{ }^{2}$ and the present study, the occurrence of exit block is associated with shorter tachycardia cycle lengths.

The present study is the first to describe the occurrence of exit block from more than one pulmonary vein during the same episode of atrial fibrillation.

\section{Limitations}

A limitation of this study is that, to minimize the duration of the procedures and the risk of complications, not all pulmonary veins were systematically mapped in every patient. Therefore, the prevalence of exit block in two or more pulmonary veins during an episode of atrial fibrillation may have been underestimated. Another limitation is that, in most patients, mapping of different pulmonary veins was performed in sequential fashion with a single catheter. Therefore, a nonsynchronized pattern of exit block was documented with simultaneous recordings in only one of the three patients in this study in whom exit block was observed in two pulmonary veins. In the other two patients, the mechanism of simultaneous tachycardias could not be definitively determined.

\section{Conclusion}

Exit block is a common observation during tachycardias generated within pulmonary veins and appears to be a reliable indicator that an arrhythmogenic pulmonary vein has been identified. The occurrence of exit block in more than one pulmonary vein at the same time probably is attributable to simultaneous tachycardias, one of which may be triggered by the other.

\section{References}

1. Jaïs P, Haïssaguerre M, Shah DC, Takahashi A, Hocini M, Quiniou G, Garrigue S, Le Mouroux A, Le Metayer P, Clementy J: A focal source of atrial fibrillation treated by discrete radiofrequency ablation. Circulation 1997;95:572-576.

2. Haïssaguerre M, Jaïs P, Shah DC, Takahashi A, Hocini M, Quiniou G, Garrigue S, Le Mouroux A, Le Metayer P, Clementy J: Spontaneous initiation of atrial fibrillation by ectopic beats originating in the pulmonary veins. N Engl J Med 1998;339:659-666.

3. Lau CP, Tse HF, Ayers GM: Defibrillation-guided radiofrequency ablation of atrial fibrillation secondary to an atrial focus. J Am Coll Cardiol 1999:33:1217-1226.

4. Chen SA, Hsieh MH, Tai CT, Tsai CF, Prakash VS, Yu WC, Hsu TL, Ding YA, Chang MS: Initiation of atrial fibrillation by ectopic beats originating from the pulmonary veins: Electrophysiological characteristics, pharmacological responses, and effects of radiofrequency ablation. Circulation 1999;100:1879-1886.

5. Hsieh MH, Chen SA, Tai CT, Tsai CF, Prakash VS, Yu WC, Liu CC, Ding YA, Chang MS: Double multielectrode mapping catheters facilitate radiofrequency catheter ablation of focal atrial fibrillation originating from pulmonary veins. J Cardiovase Electrophysiol 1999:10:136-144.

6. Lok NS, Lau CP, Ho DSW, Tang YW: Hemodynamic effects and clinical determinants of defibrillation threshold for low energy transvenous atrial defibrillation in patients with chronic atrial fibrillation. PACE 1997;20:899-908,

7. Nathan H, Eliakim M: The junction between the left atrium and the pulmonary veins: An anatomic study of human hearts. Circulation 1966:34:412-422

8. Paes de Almeida O, Bohm CM, de Paula Carvalho M, Paes de Carvalho A: The cardiac muscle in the pulmonary vein of rat: A morphological and electrophysiological study. J Morphol 1975; 135:409-433.

9. Cheung DW: Electrical activity of the pulmonary vein and its interaction with the right atrium in the guinea-pig. J Physiol 1981;314:445-456.

10. Spach MS, Barr RC, Jewett PH: Spread of excitation from the atrium into thoracic veins in human beings and dogs. Am J Cardiol 1972;30:844-854

11. Ito M, Arita M, Saeki K, Tanoue M, Fukushima I: Functional properties of sinocaval conduction. Jpn J Physiol 1967:17:174189. 
Copyright of Journal of Cardiovascular Electrophysiology is the property of Blackwell Publishing Limited and its content may not be copied or emailed to multiple sites or posted to a listserv without the copyright holder's express written permission. However, users may print, download, or email articles for individual use. 